\title{
Exosomes Secreted by Toxoplasma gondii-Infected L6 Cells: Their Effects on Host Cell Proliferation and Cell Cycle Changes
}

\author{
Min Jae Kim¹, Bong-Kwang Jung ${ }^{1}$, Jaeeun Cho, ${ }^{1,2}$, Hyemi Song ${ }^{1,2}$, Kyung-Ho Pyo ${ }^{3}$, Ji Min Lee ${ }^{3}$, \\ Min-Kyung Kim ${ }^{4}$, Jong-Yil Chai ${ }^{1,2, *}$ \\ ${ }^{1}$ Department of Parasitology and Tropical Medicine, Seoul National University College of Medicine, Seoul 03080, Korea; ${ }^{2}$ Korean Association of \\ Health Promotion, Seoul 07649, Korea; ${ }^{3}$ JE-UK laboratory of Molecular Cancer Therapeutics, Yonsei Cancer Institute, Yonsei University College of \\ Medicine, Seoul 03722, Korea; ${ }^{4}$ Korean Centers for Disease Control and Prevention, Osong 28159, Korea
}

\begin{abstract}
Toxoplasma gondii infection induces alteration of the host cell cycle and cell proliferation. These changes are not only seen in directly invaded host cells but also in neighboring cells. We tried to identify whether this alteration can be mediated by exosomes secreted by T. gondii-infected host cells. L6 cells, a rat myoblast cell line, and RH strain of $T$. gondii were selected for this study. L6 cells were infected with or without $T$. gondii to isolate exosomes. The cellular growth patterns were identified by cell counting with trypan blue under confocal microscopy, and cell cycle changes were investigated by flow cytometry. L6 cells infected with T. gondii showed decreased proliferation compared to uninfected L6 cells and revealed a tendency to stay at $\mathrm{S}$ or G2/M cell phase. The treatment of exosomes isolated from $T$. gondii-infected cells showed attenuation of cell proliferation and slight enhancement of S phase in L6 cells. The cell cycle alteration was not as obvious as reduction of the cell proliferation by the exosome treatment. These changes were transient and disappeared at $48 \mathrm{hr}$ after the exosome treatment. Microarray analysis and web-based tools indicated that various exosomal miRNAs were crucial for the regulation of target genes related to cell proliferation. Collectively, our study demonstrated that the exosomes originating from $T$. gondii could change the host cell proliferation and alter the host cell cycle.
\end{abstract}

Key words: Toxoplasma gondii, exosome, cell cycle, proliferation, L6 cell

\section{INTRODUCTION}

Toxoplasma gondii is an obligate intracellular parasite that infects a wide range of mammalian cells. When host cells are infected with T. gondii, the parasite is known to modulate the host cell cycle and cell proliferation mechanisms [1]. Previous studies showed that $T$. gondii infection induced the host cells to enter $S$ phase. These changes not only appeared in T. gondiiinfected cells but also in adjacent cells [2]. The study suggested that these changes can be mediated by soluble factors in the supernatant of the culture media. Because T. gondii invades the $S$ phase cells most efficiently, the cell cycle changes of the neighboring cells are favorable for T. gondii [3]. Other studies revealed that $T$. gondii infection caused the host cells to transit

- Received 19 March 2016, revised 26 March 2016, accepted 27 March 2016.

*Corresponding author (cjy@snu.ac.kr)

(c) 2016, Korean Society for Parasitology and Tropical Medicine

This is an Open Access article distributed under the terms of the Creative Commons Attribution Non-Commercial License (http://creativecommons.org/licenses/by-nc/3.0) which permits unrestricted non-commercial use, distribution, and reproduction in any medium, provided the original work is properly cited. through G1/S phase and arrest at G2 phase $[1,4]$.

Exosomes are extracellular vesicles that contain many proteins and miRNAs. It is known to function in intercellular communications and modulate host-parasite interactions [5]. Exosomes from parasites can be transferred to host cells and change the host immune responses [6]. In addition, exosomes from a Plasmodium falciparum-infected RBC were taken by another infected RBC and changed sexual differentiation of the intra-erythrocytic stages [7]. In T. gondii, it was reported that exosomes secreted from $T$. gondii-infected cells included many miRNAs that were different from those originating from uninfected host cells [6]. However, more detailed studies, including the effects of these exosomes on host cell cycle and proliferation, have not been performed.

In this study, we supposed that the exosomes secreted from T. gondii-infected cells have effects on host cell cycle regulation and host cell proliferation. In addition, we tried to identify changes in miRNA profiles induced by exosome treatment and to predict possible target genes using a web-search. 


\section{MATERIALS AND METHODS}

\section{L6 cells and T. gondii}

L6 rat myoblast cells were maintained in Dulbecco's modified Eagle's medium (DMEM, Welgene, Seoul, Korea) supplemented with $10 \%$ fetal bovine serum (FBS, Welgene), $4 \mathrm{mM} \mathrm{L}$ glutamine, $0.2 \mathrm{mM}$ penicillin, $0.05 \mathrm{mM}$ streptomycin (Welgene) at $37^{\circ} \mathrm{C}$ in humidified air containing $5 \% \mathrm{CO}_{2}$. The $\mathrm{RH}$ tachyzoites of $T$. gondii were maintained under in vitro condition using Vero cells. The tachyzoites were collected from the culture supernatant of infected Vero cells by serial centrifugation at 1,500 rpm for $5 \mathrm{~min}$ and 3,000 rpm for $10 \mathrm{~min}$.

\section{Cell number counting and confocal microscopy}

To investigate the cell proliferation patterns, L6 cells were inoculated in 6-well plates, and cell numbers in a well were counted after trypan blue staining at pre-determined time points. The phase-contrast images of T. gondii-infected cells were acquired with confocal microscope (FV 1000, Olympus, Japan) after fixation with $4 \%$ paraformaldehyde.

\section{Isolation of exosomes}

Exosome-depleted culture media were prepared by ultracentrifugation of DMEM with 10\% FBS at 100,000 g for $16 \mathrm{hr}$ at $4^{\circ} \mathrm{C}$ followed by filtration through a $0.22-\mu \mathrm{m}$ filter (Nalgene, Rochester, New York, USA). These media were used for isolation of exosomes. At first, $5 \times 10^{5}$ L6 cells were inoculated in $100 \mathrm{~mm}$ culture dish with exosome-depleted culture media. After 12 hr of incubation, the RH tachyzoites (multiplicity of infection; MOI $=20$ ) were added. The culture media were exchanged with fresh exosome-depleted culture media $24 \mathrm{hr}$ after $T$. gondii inoculation, thereby removing the non-invaded parasites. The exosomes of control group, i.e., L6 cells without $T$ gondii infection, were also collected by the same protocol except that PBS was added instead of RH tachyzoites.

Exosomes were isolated from the culture supernatant by differential centrifugation, which is the most widely used method. Briefly, the L6 cell culture supernatants were harvested and centrifuged at $300 \mathrm{~g}$ for $10 \mathrm{~min}$ at $4^{\circ} \mathrm{C}$. The supernatant was serially transferred to a new tube and centrifuged at 2,000 $\mathrm{g}$ for $10 \mathrm{~min}$ at $4^{\circ} \mathrm{C}$ and at $10,000 \mathrm{~g}$ for $30 \mathrm{~min}$ at $4^{\circ} \mathrm{C}$. The supernatant was then ultracentrifuged at $100,000 \mathrm{~g}$ for $70 \mathrm{~min}$ at $4^{\circ} \mathrm{C}$ with ultracentrifuge (Optima XE-100 Ultracentrifuge, Beckman Coulter, Miami, Florida, USA). The observed exosome pellets in each tube were collected together and ultracentrifuged once more at $100,000 \mathrm{~g}$ for $70 \mathrm{~min}$ at $4^{\circ} \mathrm{C}$. The final pellet was resuspended in $300 \mu \mathrm{l}$ of PBS for RNA or protein analysis. The concentration of isolated exosome was determined by NanoDrop 2000 Spectrophotometer.

\section{Flow cytometry}

L6 cells were grown in 6-well plates. Initially, $2 \times 10^{5} \mathrm{~L} 6$ cells were inoculated in exosome-depleted culture media. At $12 \mathrm{hr}$, $1 \times 10^{6} \mathrm{RH}$ tachyzoites (MOI 20) or exosomes from L6 cells with or without $T$. gondii infection were added at $100 \mu \mathrm{g} / \mathrm{ml}$. The tachyzoites were allowed to invade the cells for $24 \mathrm{hr}$, and then non-invaded parasites were washed away. The culture media containing exosomes were maintained without media change. At pre-determined time points, the cells were trypsinized and washed 3 times in $1 \mathrm{ml}$ of PBS. After centrifugation at 1,200 rpm for $5 \mathrm{~min}$, cell pellets were re-suspended in $0.3 \mathrm{ml}$ PBS. The cells were fixed by incubation for $1 \mathrm{hr}$ on ice with addition of $0.7 \mathrm{ml}$ of $70 \%$ ethanol. The fixed cell suspensions were incubated in $37^{\circ} \mathrm{C}$ for $1 \mathrm{hr}$ with RNase A. Finally, the cells were stained with propidium iodide (PI) and analyzed on a cytometer at $488 \mathrm{~nm}$.

\section{miRNA microarray}

Exosomal RNA was extracted from L6 cell-derived exosomes using miRNeasy Mini Kit (Qiagen, Hilden, Germany) according to the manufacturer's instructions. The RNA concentration was determined by NanoDrop 2000 Spectrophotometer. RNA quality for microarray was assessed by Agilent 2100 Bioanalyser (Agilent Technologies, Amstelveen, Netherlands).

The miRNA expression profiling was performed using miRCURY $^{\mathrm{TM}}$ LNA microRNA Array, 7th generation-has, mmu, and rno array (EXIQON, Vedbaek, Denmark). We used 250-1,000 ng of exosomal RNA for $\mathrm{Cy} 3$ dye labelling. Labeled samples were subsequently hybridized onto a microarray slide using a hybridization chamber kit (Agilent Technologies, Santa Clara, California, USA) and hybridization gasket slide kit (Agilent Technologies). Hybridization was performed over $16 \mathrm{hr}$ at $56^{\circ} \mathrm{C}$ followed by washing the microarray slide as recommended by the manufacturer. The microarray slides were then scanned with Agilent G2565CA Microarray Scanner System (Agilent Technologies). Scanned images were imported by Agilent Feature Extraction software version 10.7.3.1 (Agilent Technologies), and fluorescence intensities of each image were quantified using the modified Exiqon protocol and corresponding GAL files. 


\section{Target genes prediction}

Among the miRNAs identified by microarray, those showing signal intensity more than $9.0(\log 2)$ were only included in the analysis. The differentially expressed miRNAs were determined as those showing greater than 4.0-fold change of signal intensity between 2 groups, exosomal miRNAs from L6 cells with or without T. gondii infection. The predicted target genes of these miRNAs were identified using multiple miRNA target prediction web tools, miRDB (http://mirdb.org/miRDB). The functions of the target genes were analyzed by another webtool, DAVID Bioinformatics Resources 6.7 (http://david.ncifcrf.gov).

\section{Statistical analysis}

The independent $t$-test was used to compare the means between 2 groups.

\section{RESULTS}

\section{T. gondii infection resulted in decrease of L6 cell proliferation through arrest of G2/M and S phase}

The $\mathrm{L} 6$ rat myoblast cell line was selected to study interaction between the host cell and T. gondii. L6 cells are known to differentiate into myocytes under confluent condition or with special differentiation medium [8]. Experiments were performed at early stages of cell culture and under normal growth medium to maintain proliferation of L6 cells. Cells were incubated in culture medium alone or with T. gondii RH tachyzoites. Non-invaded parasites were washed away after $24 \mathrm{hr}$ of incubation. The cell numbers were counted, and the phasecontrast images were acquired at the pre-determined time points. The DNA contents were measured at $24 \mathrm{hr}$ and $48 \mathrm{hr}$ after the tachyzoite inoculation by flow-cytometry analysis of PI-stained nuclei.

T. gondii-infected L6 cells showed decreased proliferation compared to uninfected cells (Fig. 1A). The difference in cell numbers between the 2 groups appeared early after $T$. gondii inoculation and became obvious at $48 \mathrm{hr}$ post infection. After $72 \mathrm{hr}$ post infection, the total cell numbers in infected cells began to decrease. The phase-contrast images showed continuous intracellular proliferation of T. gondii (Fig. 1B, C). The cell lysis was obvious at $72 \mathrm{hr}$ post infection, explaining the decrease of the cell counting. In T. gondii-infected cells, G2/M and $S$ phase were increased; however, G0/G1 phase were decreased compared to uninfected cells. Between $24 \mathrm{hr}$ and $48 \mathrm{hr}$ post in-

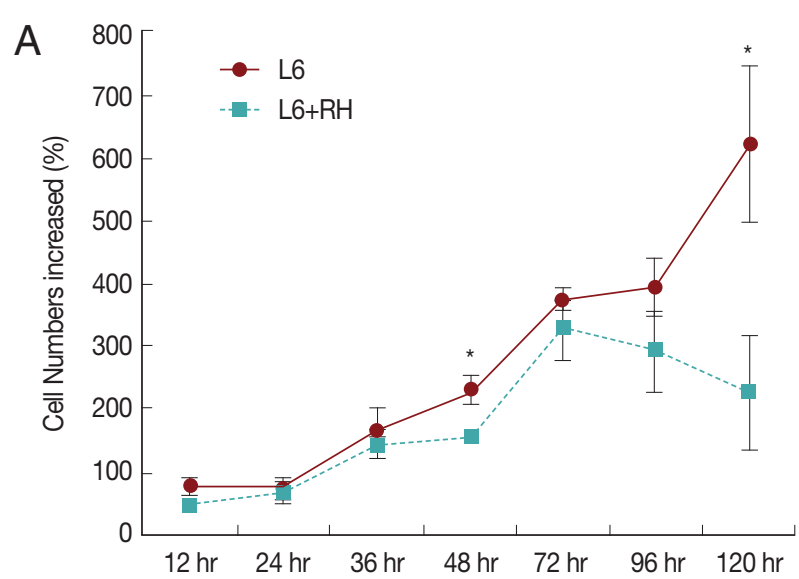

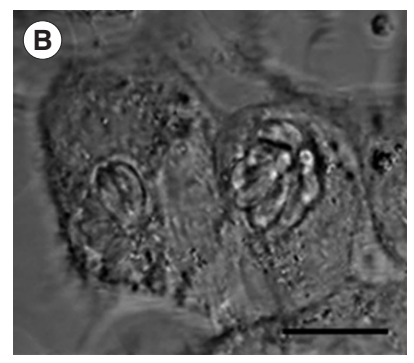

$24 \mathrm{hr}$ post infection

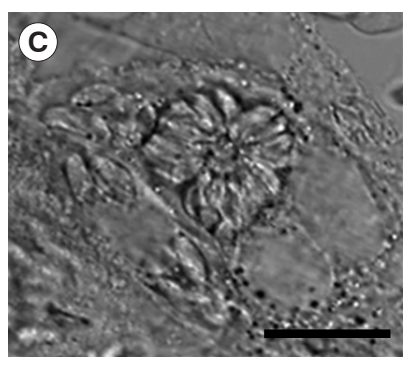

$48 \mathrm{hr}$ post infection
D

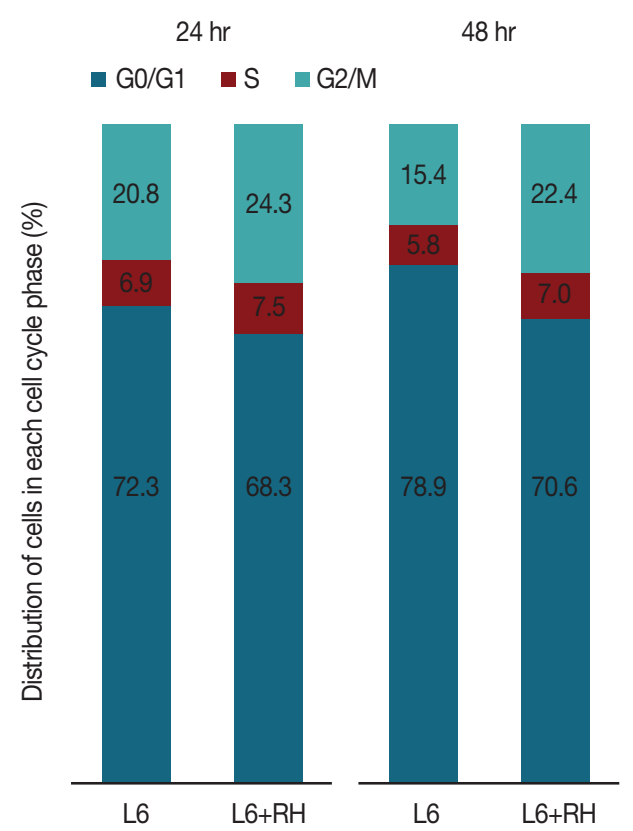

Fig. 1. (A) Cell growth patterns of uninfected and T. gondii-infected L6 cells. The cell numbers differed significantly at $48 \mathrm{hr}$ and $120 \mathrm{hr}$ post infection $\left({ }^{*} P<0.05\right)$. (B, C) Phase-contrast images of T. gondii-infected cells at $24 \mathrm{hr}$ and $48 \mathrm{hr}$ post infection $(\mathrm{MOI}=20)$, respectively. The images show continuous proliferation of $T$. gondii in host cells. Bar=10 $\mu$ m. (D) Cell cycle changes induced by T. gondii infection at $24 \mathrm{hr}$ and $48 \mathrm{hr}$ post infection. 


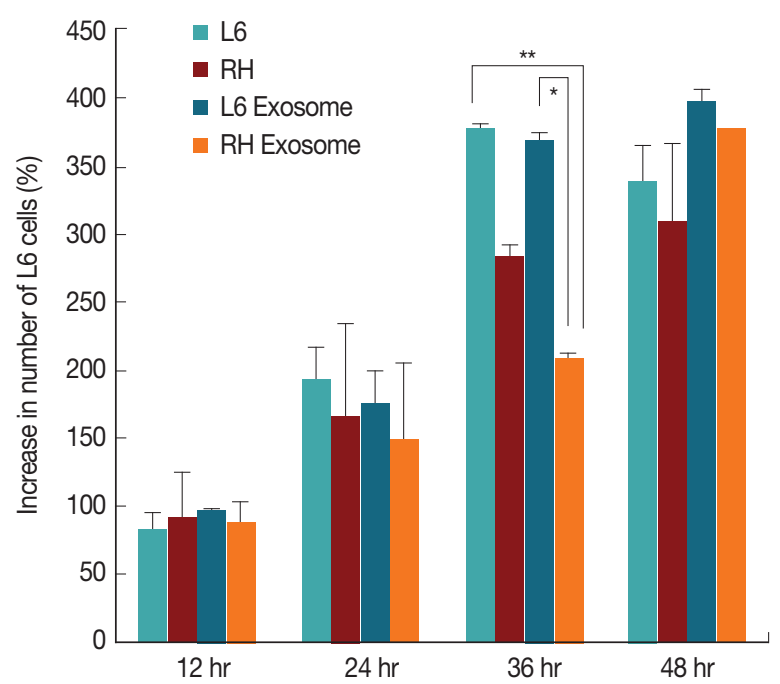

Fig. 2. Cell growth patterns of L6 cells grown with usual culture media, T. gondii-infected, treated with $100 \mu \mathrm{g} / \mathrm{ml}$ of exosomes from uninfected ( $\mathrm{L} 6$ exosome) and T. gondii-infected (RH exosome) cells. The number of L6 cells treated with $\mathrm{RH}$ exosome was significantly different from that of the cells treated with L6 exosome $\left.{ }^{*} P=0.001\right)$ or that of the cells grown with usual culture media ( $\left.{ }^{\star \star} P<0.001\right)$ at $36 \mathrm{hr}$ by independent $t$-test.

fection, the proportion of cells in the G2/M stage decreased in uninfected L6 cells from $20.8 \%$ to $15.4 \%$. However, these changes did not occur in T. gondii-infected cells (Fig. 1D).

\section{Effects of exosomes on cell cycle and cell proliferation}

To identify whether exosomes can mediate changes on cell cycle and cellular proliferation, cells were incubated with the exosomes isolated from L6 cells with or without T. gondii, respectively. Cells were trypsinized and analyzed by phase contrast microscopy for counting the cell numbers or by flow cytometry analysis for measurement of the cell cycle. All experiments were duplicated.

In L6 cells cultured with the exosomes derived from T. gondii-infected cells (considered as RH tachyzoite exosome), the host cells proliferated less than those grown in culture media alone and also those grown with the exosomes derived from uninfected L6 cells (considered as L6 cell exosome). The cells treated with RH tachyzoite exosome reached only to $208.1 \%$, whereas those treated with L6 exosome to $367.5 \%$ at $36 \mathrm{hr}$ post treatment. L6 cells without treatment with exosomes proliferated to $376.9 \%$. The difference was statistically significant at $P=0.01$ and $P<0.001$, respectively (Fig. 2). At $48 \mathrm{hr}$ post infection, these changes disappeared.

By comparison, changes in the cell cycle were not as evident as those of the cell number. Although L6 cells cultured with

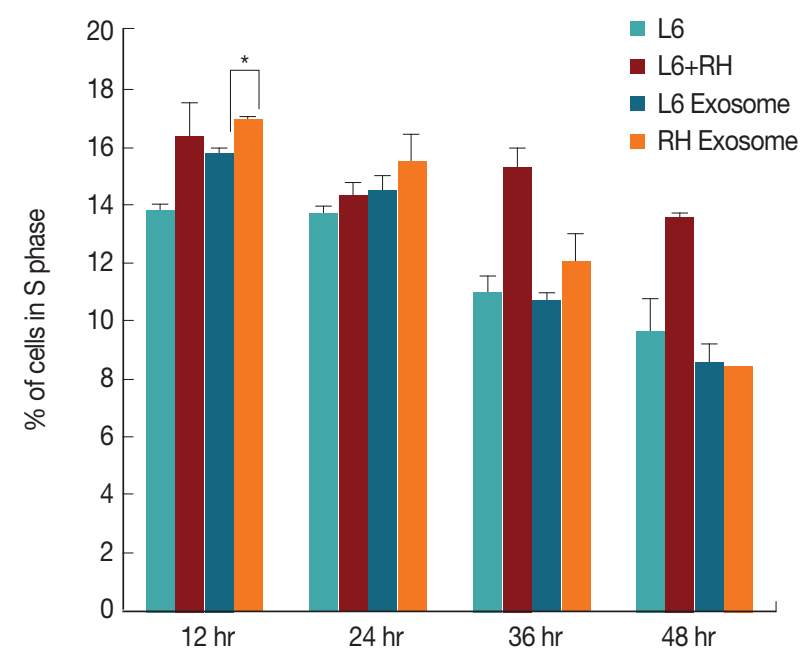

Fig. 3. Changes of the percentage (\%) of cells in the S phase at $12,24,36$, and $48 \mathrm{hr}$ post T. gondii infection or exosome treatment. Only minute difference is observed between cells treated with $\mathrm{L} 6$ exosome or $\mathrm{RH}$ tachyzoite exosome at $12 \mathrm{hr}$ post treatment $\left({ }^{*} P=0.028\right)$.

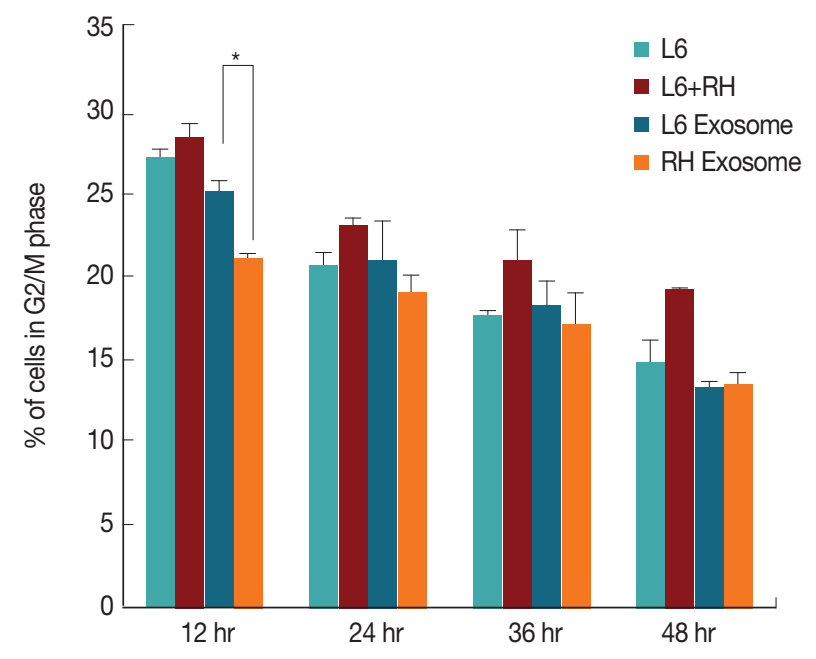

Fig. 4. Changes of the percentage (\%) of cells in the G2/M phase at $12,24,36$, and $48 \mathrm{hr}$ post $T$. gondii infection or exosome treatment. Cells treated with $\mathrm{RH}$ tachyzoite exosome showed decrease in G2/M phase compared to those treated with L6 exosome at $12 \mathrm{hr}$ post treatment ( $\left.{ }^{\star} P=0.030\right)$.

the RH tachyzoite exosome showed increase in S stage (16.9\% vs $15.8 \%, P=0.028)$, and decrease in G2/M stage ( $20.9 \%$ vs $25.0 \%, P=0.030$ ) compared to those treated with L6 exosome at $12 \mathrm{hr}$ post treatment, these changes disappeared gradually (Figs. 3, 4). At $48 \mathrm{hr}$ post infection, both cells grown with L6 cell exosome and RH tachyzoite exosome revealed similar distribution patterns on the cell cycle stage. These patterns were also similar to that of the control group, L6 cells grown only in usual culture media. 
Table 1. Eleven miRNAs with relevant gene ontology terms

\begin{tabular}{|c|c|c|c|c|}
\hline miRNA & Changes & Gene ontology from GOBPa & $P$-value & Associated genes \\
\hline rno-miR-3075 & Up-regulated & G2/M transition of mitotic cell cycle & 4.80E-02 & Khdrbs1 Ppm1d \\
\hline rno-miR-92a-2-5p & Up-regulated & Regulation of cell proliferation & 3.30E-04 & $\begin{array}{l}\text { Cebpa Fbxo2 Ihh Acvrl1 Calr Carm1 Ccnd2 Hnf4a Id3 Irs1 } \\
\text { Itga2 Ngfr Nf1 Nog Nr3c1 Nod2 Nacc1 Pemt Prkca Rac2 } \\
\text { Rarg Bcl211 S1pr1 Tp53 Vegfa Vash1 Zmiz1 }\end{array}$ \\
\hline rno-miR-1224 & Up-regulated & Regulation of cell proliferation & 4.70E-02 & Ndfip1 Acvrl1 Becn1 Camk2d Rb1 Thrb Vash2 \\
\hline rno-miR-223-3p & Up-regulated & Regulation of cell proliferation & 7.10E-03 & Pds5b Rerg F3 II6st Nfib Rps6kb1 S1pr1 Trim35 Ube2a \\
\hline rno-miR-325-3p & Up-regulated & mitotic cell cycle & 4.70E-02 & $\begin{array}{l}\text { Khdrbs1 Pds5b Cdca8 Ccng1 Foxo4 Ppm1d Slfn1 Psmc4 } \\
\text { Usp16 }\end{array}$ \\
\hline \multirow[t]{2}{*}{ rno-miR-200a-3p } & Down-regulated & Cell cycle & 2.30E-03 & Fbxo5 Pds5b Ruvbl1 Specc1l Apbb2 Cep120 Cyp26b1 \\
\hline & & & & Clasp2 Myh10 Psmd11 Ptp4a1 Siah1a Txnip Tgfb2 \\
\hline rno-miR-3072 & Down-regulated & M phase & 4.30E-02 & Cep120 Cyp26b1 Lzts2 \\
\hline \multirow[t]{2}{*}{ rno-miR-130b-5p } & Down-regulated & Regulation of growth & 2.60E-02 & Adam10 Pou3f2 Igfbp2 Lep Ptpn11 \\
\hline & & Cell cycle & 4.20E-02 & $\begin{array}{l}\text { Cep55 Ccng1 Cyp26b1 Lats2 Mtus1 Ppp6c Rbl2 Txnip } \\
\text { Ube2b }\end{array}$ \\
\hline \multirow[t]{2}{*}{ rno-miR-3586-5p } & Down-regulated & Cell cycle & 1.30E-02 & Rassf1 Rassf2 Cyp26b1 Gas2 Mnat1 Mapk13 Nusap1 \\
\hline & & & & Pafah1b1 Ppp1cc Ptp4a1 Txnip Tp73 \\
\hline rno-miR-493-3p & Down-regulated & Cell division & 2.90E-02 & Clf1 Kif3b Ralbp1 \\
\hline rno-miR-217-3p & Down-regulated & Regulation of cell proliferation & 2.80E-02 & Hipk1 II6st Nr3c1 Nod2 Rarb Tacr1 \\
\hline
\end{tabular}

${ }^{a}$ Gene ontology of biological process.

\section{miRNA microarray}

The microarray analysis was performed to investigate the difference in the levels of miRNAs contained in RH tachyzoite exosomes and L6 cell exosomes. Among the total 701 miRNAs screened, 34 miRNAs revealed increased signal intensity and 30 miRNAs showed decreased signal intensity, i.e., $>9$ normalized signal intensity and more than 4 -fold change ( $\log 2)$. The names of these significantly changed miRNAs are shown in Fig. 5.

\section{Target gene analysis}

The predicted target genes of the significantly changed miRNAs were acquired by searching web-based database, mirDB (http://mirdb.org/miRDB). Genes with target score 70 and more were selected. Gene ontology was searched via webbased tools, DAVID Bioinformatics Resources 6.7 (http://david.ncifcrf.gov) [9].

The miRNAs with gene ontology related with cell cycle or cell proliferation were searched. Only gene ontology terms with $P$-value less than 0.05 were selected. Finally, 11 miRNAs were selected, 5 of them were increased in the RH tachyzoite exosomes than in the L6 cell exosomes, and 6 decreased. These 11 miRNAs and their target genes are shown in Table 1.

\section{DISCUSSION}

We have shown that T. gondii infection of $\mathrm{L} 6$ cells resulted in decreased host cell proliferation and cell cycle alteration. L6 cells incubated with the exosomes from T. gondii-infected cells also showed similar changes, although transiently. The microarray analysis of the exosomes identified miRNAs with significant difference in signal intensity between the RH tachyzoite exosomes and the L6 cell exosomes. The overall functions of these miRNAs were estimated by gene ontology terms. Among these miRNAs, those, of which the functions suggest cell cycle and cell proliferation regulation, were also identified by webbased tools.

The cell cycle dysregulation phenomenon caused by the infectious organism is already well recognized. Many viruses alter the cell cycle of the infected host cells [10]. Among the protozoan parasites, Theileria species are famous for inducing infected cells to proliferate [11]. Several studies already showed $T$. gondii could also change the host cell cycle [4]. For example, T. gondii infection induced the G1/S transition of the human trophoblasts and fibroblasts, followed by the G2 arrest. As a result, the host cell proliferation was suppressed, and host cells at the G2/M stage increased [1]. Another study showed that $T$. gondii infection caused human fibroblasts to enter $S$ phase. This change appeared not only in directly invaded cells but also in neighboring cells. The filtered culture media from $T$. gondii-infected cell cultures caused the same changes, suggesting soluble factors mediate such parasite-host interactions [2]. These changes are regarded as beneficial for the parasite because T. gondii more easily invades cells in the $S$ stage [3]. 


rno-miR-543-5p
rno-miR-3075
rno-miR-324-5p
rno-miR-125b-1-3p
rno-miR-3074
rno-miR-183-3p
rno-miR-664-2-5p
rno-miR-411-3p
rno-miR-92b-5p
rno-miR-540-3p
rno-miR-147
rno-miR-3556a
rno-miR-291a-5p
rno-miR-200b-5p
rno-miR-miR-186-3p
rno-miR-30b-3p
rno-miR-124-3p
rno-miR-503-5p
rno-miR-465-3p
rno-miR-9b-3p
rno-miR-3p-216a-5p
rno-miR-92a-2-5p
rno-let-7d-3p
rno-miR-349a-3p
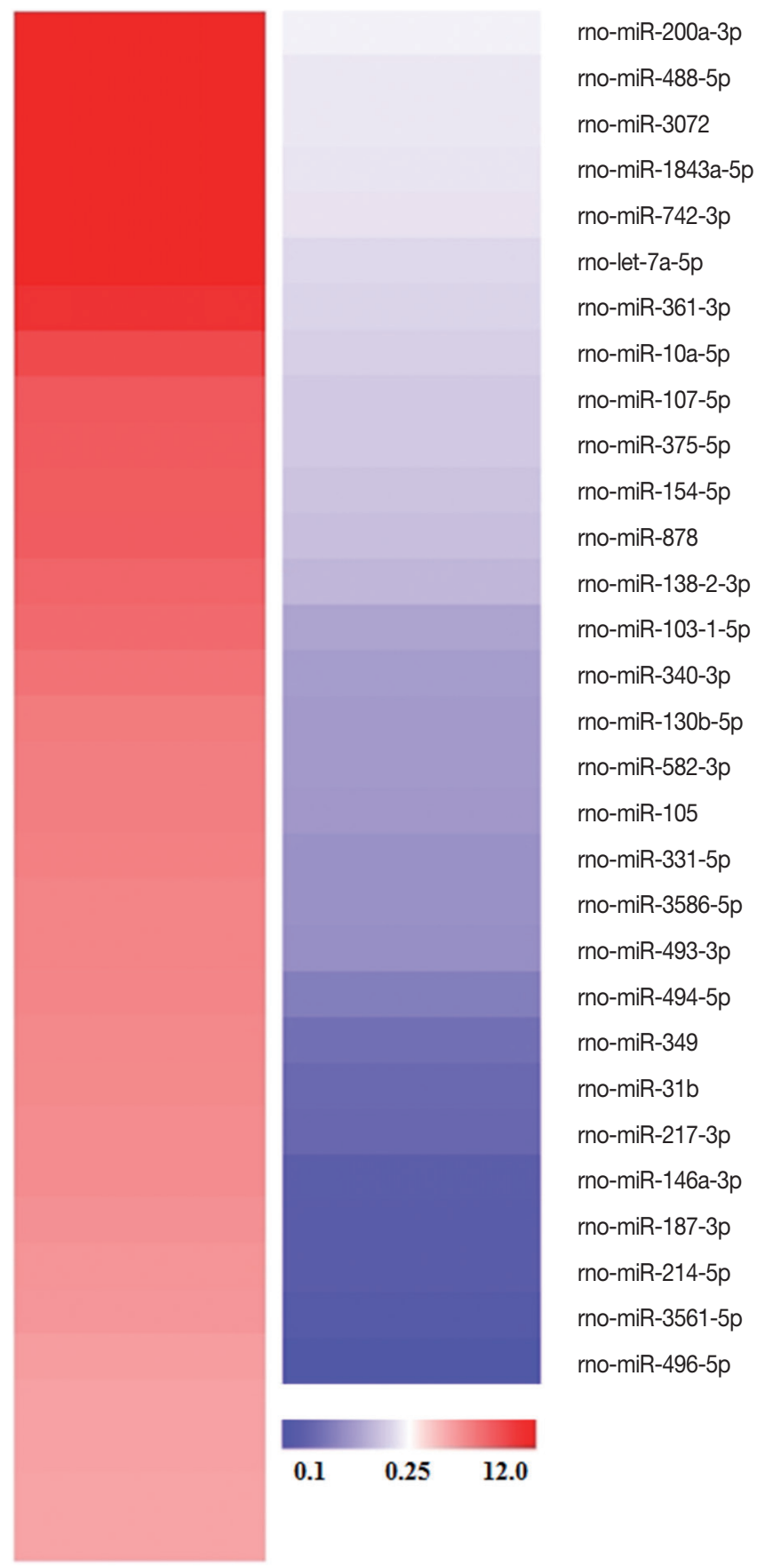

Fig. 5. Significantly changed miRNAs in T. gondii RH exosomes compared to those in L6 cell exosomes and their altered signal intensities. Among these 64 miRNAs, those which are specifically associated with cell cycle or cell proliferation are shown in Table 1.

In our study, the proportion of cells in the $S$ and G2/M phase was increased following T. gondii infection of the L6 cells. In addition, the host cell growth was suppressed compared to that of the L6 cells. The phase-contrast images of the T. gondii-infected cells showed continued intracellular replication of T. gondii at $24 \mathrm{hr}$ and $48 \mathrm{hr}$ post infection. The cell lysis was evident from $72 \mathrm{hr}$ post infection and thereafter. Thus, the decreased cell growth in the early period till $48 \mathrm{hr}$ probably represents suppressed proliferation of directly invaded cells and neighboring cells rather than cell lysis. The declining in cell number after $72 \mathrm{hr}$ post infection is thought to be associated with cell lysis. 
To verify our hypothesis that the exosomes can mediate these functional alterations in cell growth, we directly added the RH tachyzoite exosomes and the $\mathrm{L} 6$ cell exosomes in the exosome-depleted culture media and observed cell growth patterns and cell cycle changes. The uninfected $\mathrm{L} 6$ cells grown with the RH tachyzoite exosomes exhibited similar growth patterns with the T. gondii-infected L6 cells. The cell numbers were significantly lower than that of the L6 cells grown in culture media only or with the L6 cell exosomes. These differences in cell number were evident at $36 \mathrm{hr}$ after the exosome treatment and disappeared at $48 \mathrm{hr}$. Contrast to these differences in cell number, the cell cycle status did not differ much among the L6 cells grown with RH tachyzoite exosomes, L6 cell exosomes, and culture media only. Only a minute increase of $S$ phase was observed in cells grown with RH tachyzoite exosomes at $12 \mathrm{hr}$ after addition of the exosome. However, the difference was soon weakened and disappeared at $48 \mathrm{hr}$ after the exosome treatment.

We could observe not only T. gondii infection itself but also the exosomes derived from the infected cells caused the alterations in cell growth, i.e., suppression of proliferation and the tendency to stay in $\mathrm{S}$ or G2/M phase. These changes were only transient. Alterations in the cell cycle appeared early, if any, and those in the cell proliferation appeared more lately as 36 $\mathrm{hr}$ after the exosome addition. Considering that exosomes act to negatively modulate the mRNA of the target genes, we postulated that the changes in the mRNA and protein profiles occur in very early period, and later the cell cycle mechanism changes and finally the cell number differed as a final phenotype. Considering that the doubling time of the L6 cells is about $22 \mathrm{hr}$ [12], the changes which appeared at $36 \mathrm{hr}$ can be the results of actions of exosomes in early period as before $12 \mathrm{hr}$.

In contrast to the effects of direct infection of $T$. gondii, the effects of exosome treatment was only transient. When the L6 cells were infected by T. gondii, probably the infected cells continuously secreted the RH tachyzoite exosomes. In contrast, the uninfected L6 cells grown with RH tachyzoite exosomes were exposed to the exosomes only in the early period. As cells continue to proliferate, the initial environment with high quantities of RH tachyzoite exosomes would have been replaced by the exosomes secreted from the uninfected cells. This probably could explain the transient effects of the exosomes shown in this study.

Finally, we needed to find out whether the miRNAs that can alter the cell proliferation are really contained in the exosomes.
Using the microarray analysis, total 701 miRNAs were screened, and finally 64 miRNAs with significantly changed signal intensity were identified. Among them, 11 miRNAs had relevant gene ontology terms regarding cell proliferation or cell cycle. A total of 91 genes were revealed to be regulated by these 11 miRNAs. Among them, there existed famous cell cycle regulatory genes, such as cyclin D2 (Ccnd2) and retinoblastoma 1 (Rb1) [13]. Genes associated with sister chromatid segregation (PDS5 cohesin associated factor B; Pds5b), chromosome movement (kinensin family member 3B; Kif3b), or centrosome functions (centrosomal protein $120 \mathrm{kDa}$ and centrosomal protein $50 \mathrm{kDa}$; Cep120 and Cep55) are also included [14-16]. Some genes were functioned in the p53 signaling pathway (homeodomain interacting protein kinase 1; Hipk1) and some others were in the TGF $\beta$ pathway (activin A receptor like type 1; Acvrl1) [17,18].

These target genes were identified using the nucleotide sequences of the miRNAs and web-based database. Thus, whether these miRNA-gene interactions certainly happen is still needed to confirm. However, we could suggest the possible miRNAs and their target genes.

In the previous studies using the exosomes derived from $T$. gondii infected cells, the immunological properties of the exosomes were mainly investigated. Cells with immunological functions, such as dendritic cells or macrophages, were usually used in the experiment. The effects of exosomes on inflammatory responses and immunological protection were usually investigated [19-21]. In contrast, we focused on the cell to cell communication as a function of exosomes in $T$. gondii infection.

In conclusion, we identified that $T$. gondii infection alters the cell proliferation mechanisms of the L6 cells and that the exosomes secreted by $T$. gondii-infected cells can mediate such changes to neighboring cells. Microarray analyses of the exosomal miRNAs suggested that miRNAs contained in the exosomes could have a possible role. Further studies are needed to confirm the actual role of each miRNA and their target genes in regulation of the host cell cycle and proliferation.

\section{CONFLICT OF INTEREST}

We have no conflict of interest related to this study.

\section{REFERENCES}

1. Brunet J, Pfaff AW, Abidi A, Unoki M, Nakamura Y, Guinard M, 
Klein JP, Candolfi E, Mousli M. Toxoplasma gondii exploits UHRF1 and induces host cell cycle arrest at G2 to enable its proliferation. Cell Microbiol 2008; 10: 908-920.

2. Lavine MD, Arrizabalaga G. Induction of mitotic S-phase of host and neighboring cells by Toxoplasma gondii enhances parasite invasion. Mol Biochem Parasitol 2009; 164: 95-99.

3. Grimwood J, Mineo JR, Kasper LH. Attachment of Toxoplasma gondii to host cells is host cell cycle dependent. Infect Immun 1996; 64: 4099-4104.

4. Molestina RE, El-Guendy N, Sinai AP. Infection with Toxoplasma gondii results in dysregulation of the host cell cycle. Cell Microbiol 2008; 10: 1153-1165.

5. Coakley G, Maizels RM, Buck AH. Exosomes and Other Extracellular Vesicles: The New Communicators in Parasite Infections. Trends Parasitol 2015; 31: 477-489.

6. Buck AH, Coakley G, Simbari F, McSorley HJ, Quintana JF, Le Bihan T, Kumar S, Abreu-Goodger C, Lear M, Harcus Y, Ceroni A, Babayan SA, Blaxter M, Ivens A, Maizels RM. Exosomes secreted by nematode parasites transfer small RNAs to mammalian cells and modulate innate immunity. Nat Commun 2014; 5: 5488.

7. Regev-Rudzki N, Wilson DW, Carvalho TG, Sisquella X, Coleman BM, Rug M, Bursac D, Angrisano F, Gee M, Hill AF, Baum J, Cowman AF. Cell-cell communication between malaria-infected red blood cells via exosome-like vesicles. Cell 2013; 153: 11201133.

8. Lawson MA, Purslow PP. Differentiation of myoblasts in serumfree media: effects of modified media are cell line-specific. Cells Tissues Organs 2000; 167: 130-137.

9. Huang da W, Sherman BT, Lempicki RA. Systematic and integrative analysis of large gene lists using DAVID bioinformatics resources. Nat Protoc 2009; 4: 44-57.

10. Bagga S, Bouchard MJ. Cell cycle regulation during viral infection. Methods Mol Biol 2014; 1170: 165-227.

11. von Schubert C, Xue G, Schmuckli-Maurer J, Woods KL, Nigg EA, Dobbelaere DA. The transforming parasite Theileria co-opts host cell mitotic and central spindles to persist in continuously dividing cells. PLoS Biol 2010; 8.

12. D'Amore T, Lo TC. Properties of hexose-transport regulatory mutants isolated from L6 rat myoblasts. Biochem J 1988; 250: 5964.

13. Vermeulen K, Van Bockstaele DR, Berneman ZN. The cell cycle: a review of regulation, deregulation and therapeutic targets in cancer. Cell Prolif 2003; 36: 131-149.

14. Shintomi K, Hirano T. Releasing cohesin from chromosome arms in early mitosis: opposing actions of Wapl-Pds 5 and Sgo1. Genes Dev 2009; 23: 2224-2236.

15. Haraguchi K, Hayashi T, Jimbo T, Yamamoto T, Akiyama T. Role of the kinesin-2 family protein, KIF3, during mitosis. J Biol Chem 2006; 281: 4094-4099.

16. Kumar A, Rajendran V, Sethumadhavan R, Purohit R. CEP proteins: the knights of centrosome dynasty. Protoplasma 2013; 250: 965-983.

17. Rey C, Soubeyran I, Mahouche I, Pedeboscq S, Bessede A, Ichas F, De Giorgi F, Lartigue L. HIPK1 drives p53 activation to limit colorectal cancer cell growth. Cell Cycle 2013; 12: 1879-1891.

18. Roman BL, Pham VN, Lawson ND, Kulik M, Childs S, Lekven AC, Garrity DM, Moon RT, Fishman MC, Lechleider RJ, Weinstein BM. Disruption of acvrl1 increases endothelial cell number in zebrafish cranial vessels. Development 2002; 129: 3009-3019.

19. Beauvillain C, Ruiz S, Guiton R, Bout D, Dimier-Poisson I. A vaccine based on exosomes secreted by a dendritic cell line confers protection against $T$. gondii infection in syngeneic and allogeneic mice. Microbes Infect 2007; 9: 1614-1622.

20. Aline F, Bout D, Amigorena S, Roingeard P, Dimier-Poisson I. Toxoplasma gondii antigen-pulsed-dendritic cell-derived exosomes induce a protective immune response against $T$. gondii infection. Infect Immun 2004; 72: 4127-4137.

21. Bhatnagar S, Shinagawa K, Castellino FJ, Schorey JS. Exosomes released from macrophages infected with intracellular pathogens stimulate a proinflammatory response in vitro and in vivo. Blood 2007; 110: 3234-3244. 